\title{
Use of reverse transcriptase loop-mediated isothermal amplification assay for field detection of Newcastle disease virus using less invasive samples
}

\author{
H Kirunda ${ }^{* 1}$, O M M Thekisoe ${ }^{2}$, P D Kasaija ${ }^{1}$, S D Kerfua ${ }^{1}$, G W Nasinyama ${ }^{3}$, J Opuda-Asibo ${ }^{4}$, N Inoue ${ }^{5}$
}

1. National Livestock Resources Research Institute (NaLIRRI), P.O Box 96, Tororo (Uganda); 2. Department of Zoology and Entomology,University of the Free State - Qwaqwa Campus Private Bag X13, Phuthaditjhaba 9866 (South Africa) 3. Department of Veterinary Public Health and Preventive Medicine, School of Veterinary Medicine, Makerere University, P.O Box 7062 (Uganda); 4. Office of the

Deputy Vice Chancellor (Academic Affairs) Kyambogo University, P.O Box 1, Kyambogo (Uganda);

5. National Research Center for Protozoan Diseases, Obihiro University of Agriculture and Veterinary Medicine, Obihiro, Hokkaido, 080-8555 (Japan)

* Corresponding author email: halidkirunda@gmail.com

Received: 18-10-2011, Accepted: 04-11-2011, Published Online: 30-12-2011

doi:10.5455/vetworld.2012.206-212

\begin{abstract}
A novel nucleic acid amplification method, loop-mediated isothermal amplification, was developed and recently demonstrated detection of Newcastle disease virus (NDV) in tissue samples. But slaughter of poultry for test samples is often faced with resentment by low-income farmers. This study was undertaken to determine the test properties of reverse transcriptase loop-mediated isothermal amplification (RT-LAMP) in detection of NDV in clinical cases using cloacal and oropharyngeal swabs. Samples included 46 tracheal tissues, 94 cloacal and 107 oro-pharyngeal swabs from on-station and 30 spleens, 74 cloacal and 74 oro-pharyngeal swabs from the field. Analysis was done using specific RT-LAMP targeting the fusion (F) protein. While the method detected NDV from swab samples, no RNA of other poultry disease viruses was amplified, indicating analytical specificity of $100 \%$. RT-LAMP took $\leq 36$ minutes in $83 \%(n=329)$ of positive reactions with all samples amplified in $<60$ minutes. Results were easily observed with a naked eye. Cloacal and oro-pharyngeal swabs could be a convenient and cheaper alternative in diagnosis of NDV infection by RT-LAMP in resource poor countries.
\end{abstract}

Keywords: Newcastle disease virus; RT-LAMP; sensitivity; specificity; swab sample

To cite this article : Kirunda H, Thekisoe OMM, Kasaija PD, Kerfua SD, Nasinyama GW, Opuda-Asibo J, Inoue N (2012) Use of reverse transcriptase loop-mediated isothermal amplification assay for field detection of Newcastle disease virus using less invasive samples, Vet. World. 5(4): 206-212, doi: 10.5455/vetworld.2012.206-212.

\section{Introduction}

Newcastle disease (ND) is the principal factor limiting rural poultry production in Asian and African countriesincluding Uganda. The disease may kill up to $80 \%$ of household poultry and is caused by enveloped RNA viruses of the Avian Paramyxovirus serotype 1 (APMV-1) (Alexander 2003). To prevent spread of Newcastle disease virus (NDV) during outbreaks, faster methods of diagnosis are required (IAEA/FAO, 2006). Virus isolation is the gold standard method for detection of NDV, although haemagglutination inhibition (HI) test is considered the standard laboratory test for the diagnosis of ND (OIE, 2010).

In Uganda, diagnosis of ND is often based on clinical history, clinical signs, lesions and laboratory tests mainly Haemagglutination (HA) and immunochromatographic assay (ICA) strips. Diagnosis by reverse transcriptase polymerase chain reaction (RT-
PCR) is also gradually being introduced. HI test is labour-intensive requiring serial dilution of serum samples, standardization of viruses and manual reading of plates (Rothbarth et al., 1999). The test needs two samples, with atleast two weeks interval to differentiate between antibodies due to vaccination or active infection (Blomstrom et al., 2008). Similarly, the requirements for running RT-PCR including reaction time of at least 90 minutes make RT-PCR labourious, time consuming and expensive (Rychlik et al.,1990; Pavlov et al., 2004). About a decade ago, a novel nucleic acid amplification method called loopmediated isothermal amplification (LAMP) was developed (Notomi et al., 2000). The method can amplify a tiny amount of either Deoxyribonucleic acid (DNA) or Ribonucleic Acid (RNA) rapidly and specifically under isothermal conditions with high sensitivity and specificity (Nagamine et al., 2002a).

This method is performed using the Bst DNA 
polymerase large fragment and requires four or six primers targeting six or eight distinct sequences, respectively, on the target gene. Recently, it was demonstrated that the LAMP-based assay can detect NDV in tissue samples rapidly and with high sensitivity and specificity (Pham et al., 2005). Since development of more convenient and rapid methods for the diagnosis of diseases is being encouraged (IAEA/FAO, 2006), this study was conducted to assess the potential of LAMP in detection of NDV from cloacal and oro-pharyngeal swabs in clinical cases of experimental and natural infection.

\section{Materials and Methods}

Ethical Considerations: This work was approved by the Faculty Higher Degrees, Research and Ethical Committee of the Faculty of Veterinary Medicine, Makerere University and animal experimental work undertaken at National Livestock Resources Research Institute in Uganda.

Experimental chickens: A total of 230 male layer day-old chicks obtained from a commercial breeding farm, Bokomo Breeders Company (Uganda) were used in an on-station experiment. Chicks were introduced at day 1 of age, kept under biosecure conditions and fed on commercial feeds. No vaccination was done, treatment only involved use of coccidiostats and a decline in maternal antibodies against ND was monitored for up to five weeks. Blood was collected from a cumulative total of 82 randomly selected chicks at a proportion of $10 \%$ of the flock weekly for five weeks. Serum was tested for antibodies titres against ND by HI test (OIE, 2010).

Virus isolates: Sixteen velogenic NDV isolates from Eastern Uganda (Otim et al., 2004) were used for experimental infection. Virus passaging and HA test were done as described in the Manual of Diagnostic Tests and Vaccines for Terrestrial Animals 2010 (OIE, 2010).

Virus inoculation: At five weeks of age, 138 chicks were inoculated with $0.3 \mathrm{ml}$ of a $10 \%$ diluted NDVladen allantoic fluid by intranasal and ocular routes as earlier on described (Steel et al., 2009). Chicks were then monitored for clinical signs. Ten chicks (not inoculated) formed the control group.

Sample collection: Tracheal tissues, cloacal and oropharyngeal swabs were collected at peak of clinical signs. Collection of blood and postmortem examination were conducted as described by Hofstad et al. (1984) and Fowler (1996). Forty six tracheal tissues, 94 cloacal and 107 oro-pharyngeal swabs were taken from experimental chicks while field samples included 30 spleen tissues, 74 cloacal and 74 oropharyngeal swabs. Clinical signs and lesions as described in Calnek et al. (1997) and ICA were used to confirm NDV infection before sample collection. ICA (Anigen Rapid NDV Ag Test $\mathrm{Kit}^{\circledR}$, Animal Genetics, Inc., Korea) was used according to manufacturer's instructions (www.anigen.co.kr.com). Positive test control samples included five each of the bursa of fabricius and lungs for infectious bursal disease virus (IBDV) and infectious bronchitis virus (IBV), respectively, and spleens from the control group for host chicken RNA.

RNA extraction: The genomic viral RNA from tracheal/spleen tissues, cloacal/oro-pharyngeal swabs was extracted using RNeasy® Mini kit and the QIAamp® Viral RNA Mini Kit, respectively (Quagen Inc., Valencia, CA, USA), and stored in RNAase free cryogenic vials (Ambion, Applied Biosystems, Woodward, USA). The eluted RNA, previously stored at $-80{ }^{\circ} \mathrm{C}$, was later precipitated using the ethanol precipitation of nucleic acids procedures (http://open wetware.org/wiki/Ethanol_precipitation_of_nucleic_ acids). The method involved the use of absolute ethanol $(99.7 \%$ ethanol), sodium acetate and refrigerated centrifuge. Precipitation was undertaken in order to facilitated shipment of RNA samples at a temperature of about $20^{\circ} \mathrm{C}$ to $22^{\circ} \mathrm{C}$ to Obihiro University of Agriculture and Veterinary Medicine, Japan, where RT-LAMP analysis was later done after re-dissolving in sterile RNAase-free double-distilled water, vortexed and span down to re-suspend.

RT-LAMP analysis: The specific oligonucleotide primers used were designed for the NDV conserved region using NDV strain ASTR/74 (Accession number: Y19016) to target the complete genome of the fusion $(\mathrm{F})$ protein. The fusion ( $\mathrm{F}$ antigen) protein is the most important glycoprotein in pathogenicity and virulence of the NDV as it provides the penetration function for the virus particle and is also required for fusion between infected and adjacent non-infected cells (Nagai et al., 1989). Primer design was done using primer explorer V4 software (http:// primerexplorer.jp/elamp4.0.0/index.html). Sequences of the primer set (named UGA2 in this study) are:

FIP-5'CGGGTCCTCACCCAAAGATGTTGGTG
GAAAGCGCGTACAG-3';
BIP-5'ACACTCATGGGGGCCGAAGGTGAAGA
CCCTCGTTGGTACA3';


F3-5'CAAGCCTGGGCGGTTC3'; B3-5'ACAATAAGGCGGGGGAGAA3'; LF-5' ACACTTTGATGGATAAGATAAC3' and LB-5'CAGAGTTCTCACAGTAGGGA3'.

Primers were first optimised at different reaction temperatures ranging from $60{ }^{\circ} \mathrm{C}-65^{\circ} \mathrm{C}$ for 90 minutes. Specificity tests were conducted against poultry RNA viruses known to infect chickens in Uganda including IBDV and IBV, and host chicken RNA. Loopamp® RNA amplification kit (Eiken Chemical Co, Tokyo, Japan) was used in all RT-LAMP reactions and incubation done on a heat block or in a turbidimeter. In cases where visualization was to be done under ultra violet light, a fluorescent detection reagent (FD) (Eiken Chemical Co., Ltd.) was added into the reaction tubes. The heat block was a thermocycler (GeneAmp, PCR System 9700, Applied Biosystem, Singapore), which was set at $64{ }^{\circ} \mathrm{C}$ for 60 minutes (amplification time) and $80{ }^{\circ} \mathrm{C}$ for three minutes (to terminate the elongation and entire reaction) and held at $4{ }^{\circ} \mathrm{C}$ for short-term storage. Conversely, a real-time LAMP turbidimeter (Loopamp LA 200, Teramics, Japan) was the other incubation equipment used. Reaction products from both methods were read immediately after termination of each reaction. Results from the heat block were visualised by the naked eye based on developed turbidity or colour change of positive reactions which turned green under ultra violet light whilst negative reactions remained orange. Results of turbidimetry were read by use of generated melting curves (Mori et al., 2004).

Determination of relative diagnostic sensitivity and specificity: Relative diagnostic sensitivity (Se) and specificity (Sp) of RT-LAMP of cloacal and oropharyngeal swabs were determined by comparing the test using each of the sample categories against ICA (Martin et al., 1987). A kappa statistic was calculated to determine the level of agreement (Martin et al., 1987) and in each case, this was done after determining whether or not a test bias existed. Existence of a test bias was assessed by a non-parametric statistic, McNemar's test (Motulsky, 1995).

\section{Results}

Clinical signs among infected chicks: On day 3, post-inoculation chicks started showing signs of NDV infection as described by Calnek et al. (1997) with morbidity and mortality rates of $100 \%$ and $93.5 \%$, respectively, had been recorded by the end of the fifth day of clinical disease. Only nine out of the 138 infected chicks survived. ND lesions (Calnek et al., 1997) were observed in $90.5 \%(n=63)$ of internal organs in cases in which postmortem was done. All chicks in the control group did not show signs or lesions of ND during the experimental period and none had died by time of termination of study.

Analytical specificity and sensitivity of RTLAMP: Using a primer set (UGA 2) at an optimal reaction temperature of $64{ }^{\circ} \mathrm{C}$, NDV RNA was amplified in 20 to 36 minutes in three repetitions.

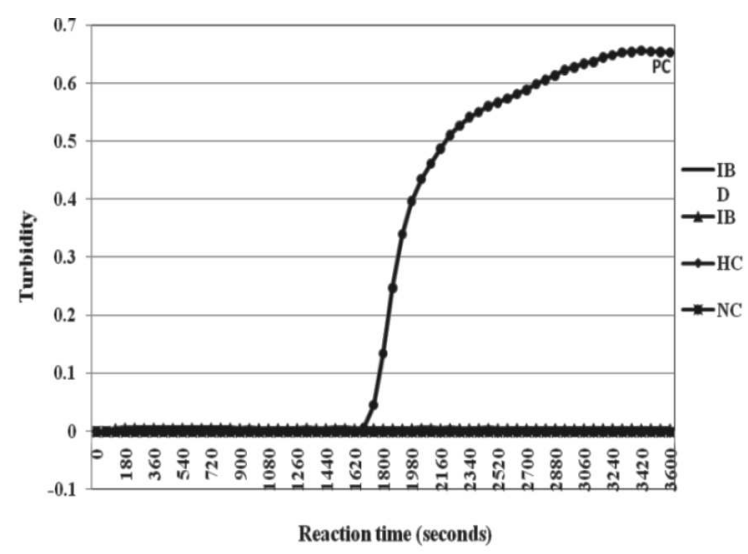

Fig. 1. Specificity of RT-LAMP with Newcastle Disease Virus ribonucleic with UGA2 primer set

IBD - Infectious bursal disease viral RNA; IB - Infectious bronchitis viral RNA; HC - Host RNA (Chicken); NC Negative non RNA control (Distilled Water); and PC Positive Control (Newcastle Disease Viral RNA)

The primer set specifically amplified the NDV RNA but did not amplify RNA of infectious bursal disease virus (IBDV), infectious bronchitis virus (IBV) or that of host chicken. The primers neither amplified RNA free water (Fig. 1).

RT-LAMP detection of Newcastle disease virus in experimental samples: Of the 46 RNA samples from tracheal tissues, $45(97.8 \%)$ were positive just as 93/94 (98.9\%) and 106/107 (99.1\%) from cloacal and oro-pharyngeal swabs, respectively. A deliberate comparison of results of RT-LAMP made for 37 sets of samples, each of cloacal and oro-pharyngeal collected from randomly selected chicks among those with prominent ND signs produced similar results. It was observed that RNA amplification achieved in agreement in $100 \%$ of sets of swabs.

RT-LAMP detection of New castle disease virus in field samples: There was variation in results when 74 cloacal and 74 oro-pharyngeal clinical samples were 
Table-1. Newcastle disease virus detection by haemagglutination test and RT-LAMP among field clinical samples

\begin{tabular}{lcccc}
\hline Sample Type & Number Tested & \multicolumn{2}{c}{ Proportions (\%) of Positive Results } \\
\cline { 2 - 4 } & & ICA & Turbidimeter & Naked eye \\
\cline { 2 - 4 } & & $97.30 \%$ & $100 \%$ & $97.30 \%$ \\
Cloacal Swabs & 74 & $94.60 \%$ & $98.60 \%$ & $97.30 \%$ \\
Oro-pharyngeal Swabs & 5 & $0.00 \%$ & $0.00 \%$ & $0.00 \%$ \\
Bursa of fibricius from IBD\# Clinical Cases & 5 & $0.00 \%$ & $0.00 \%$ & $0.00 \%$ \\
Lungs from IB+ Clinical Cases & 5 & $0.00 \%$ & $0.00 \%$ & $0.00 \%$ \\
Spleens of negative control chicks & 6 & $0.00 \%$ & $0.00 \%$ & $0.00 \%$ \\
Distilled Water & 5 & & \\
\hline
\end{tabular}

$\mathrm{ICA}^{*}=$ I mmunochromatographic Assay, RT-LAMP** = Reverse transcriptase loop-mediated

Table- 2. Relative sensitivity and specificity of RT-LAMP using cloacal and oro-pharyngeal swabs

\begin{tabular}{lccccl}
\hline Sample Category & Relative Sensitivity & Relative Specificity & McNemar's Statistic & Conclusion & Kappa (k) \\
\hline Cloacal Swabs & $100 \%$ & $33 \%$ & 4 & Test Bias Exits & $\begin{array}{l}\text { Due to test bias calculating } \\
k \text { is of limited value }\end{array}$ \\
Oro-pharyngeal Swabs & $100 \%$ & $67 \%$ & 2 & No test bias & 0.1 (No test agreement) \\
\hline
\end{tabular}

RT-LAMP = Reverse transcriptase loop-mediated

analysed using ICA, a real-time turbidimeter and by a heat block. In Table 1, results of ICA, RT-LAMP by turbidimeter and a heat block are shown. Whereas positive results of cloacal swabs by both ICA and RTLAMP (with a naked eye) were similar (97.3\%), these were however lower than $100.0 \%$ positive results observed in RT-LAMP (using a turbidimeter). Conversely, results of oro-pharyngeal samples by RTLAMP deduced by a naked eye were lower $(97.3 \%)$ than those of RT-LAMP turbidimetry $(98.6 \%)$, though higher than those of ICA (94.6\%). Results of samples from non-ND samples (IBDV and IBV) and those from healthy host chickens were similar $(0.0 \%)$ for all three tests. RNA of all 30 (100\%) spleen samples from field clinical cases was positive by RT-LAMP on either heat block or turbidimeter (results not shown). In another comparison, RT-LAMP detected NDV in all $(100 \%, n=74)$ cloacal samples taken from all ND clinical cases regardless of age group while the detection proportion of ICA was only $96 \%$ (results not shown), specifically lower in chicks than adult chickens.

Relative sensitivity and specificity of RT-LAMP using less invasive samples: A comparison of NDV detection by ICA with either of RT-LAMP for cloacal swabs or oropharyngeal swabs had relative sensitivity/specificity of $100 \% / 33 \%$ for cloacal swabs and $100 \% / 67 \%$ for oro-pharyngeal swabs, respectively (Table-2). Calculating the level of agreement $(\kappa)$, was of limited value due existence of bias in the comparison of ICA with RT-LAMP using cloacal swabs. Due to absence of test bias, $\kappa$ of 0.1 was computed for RTLAMP using oro-pharyngeal swabs, which indicted absence of agreement between the two tests.

\section{Discussion}

ND is an economically important disease affecting chickens, particularly in developing countries including Uganda (Mukiibi, 1992), is still endemic in the country (Otim et al., 2007) and chickens in many villages remain fully susceptible to infection with virulent NDV (Mukiibi-Muka and Olaho-Mukani, 1998). Effective vaccines against NDV infections have been developed and are widely applied for prevention of the disease worldwide in both commercial and free ranging production systems (Thekisoe et al., 2004). However, accurate screening of samples before vaccination and during outbreaks is of paramount importance. Whereas the use of clinical signs, haemaglutination inhibition and chromatographic tests are widely applied to confirm the presence or absence of NDV infections, development of more convenient and rapid methods for the diagnosis of diseases is being encouraged (IAEA/FAO,2006).

The RT-LAMP assay targeting the F protein of the Ugandan strain of NDV specifically amplified the RNA of the virus and amplified neither RNA of other avian viral infections commonly occurring chickens in the country including IBDV and IBV nor RNA from un-infected host chicken. A field sample detection rate of $>99 \%$ and $>97 \%$ of RT-LAMP by turbidimetry and with a naked eye, respectively, were fair for swab samples. Unlike the study by Pham et al.(2005) in which no NDV detection was made from spleen samples, in this study RT-LAMP amplified RNA from 
spleen tissues in all $(100 \%, \mathrm{n}=30)$ reactions. This result was not surprising since internal organs including spleen have been reported of a good viral load during infection with velogenic NDV, especially up to 7-10 days post infection (Kommers et al., 2002; Okwor, 2007). Detecting NDV in spleen could most likely be attributed to strain difference in tissue tropism of the velogenic (used in our study) compared with the lentogenic strain that was used by Pham et al. (2005). The good detection rates achieved with cloacal and oro-pharyngeal swabs demonstrated good RNA recovery by the two specimen categories. Swabs produced good RNA recovery rate because they were taken at the most appropriate time (about 2-5 days) post-infection, at peak of clinical status (Wakamatsu et al., 2006). LAMP products from cloacal and oro-pharyngeal samples have also demonstrated the ease of distinguishing between positive and negative reactions based on turbidity or colour change using the naked eye immediately after the reaction. Ease of differentiating positive from negative samples after a LAMP reaction with a naked eye or under ultra violet light has been extensively reported in the recent past (Tomita et al., 2008). RT-LAMP assay amplified samples obtained both in experimental infection and natural exposure within 60 minutes at a constant isothermal reaction temperature of $64^{\circ} \mathrm{C}$ using simple heating devices.

The LAMP assay is, therefore, rapid and simpler to use than RT-PCR, since the later requires thermal profiling and at least 90 minutes to run a reaction (Rychlik et al., 1990; Pavlov et al., 2004). Cloacal and oro-pharyngeal swabs, which are less invasive samples and are convenient to transport to testing stations, can be used as specimens in early diagnosis of NDV infection by this method. The relative sensitivity of $100 \%$ and $100 \%$ reported of RT-LAMP using cloacal and oro-pharyngeal swabs, respectively, reported in our study provide evidence that RT-LAMP using the two sample types is good when compared with ICA. The level of relative diagnostic sensitivity in both cases was higher than $95.5 \%$ and $96.8 \%$ reported of the method in detection of IBDV and NDV in related studies by Xue et al., (2009) and Li et al., (2009), respectively. The higher level of relative sensitivity could have been due to the fact all samples in our study were collected during day 3-5 when virus dissemination is expected to be at peak in chickens post-infection (Wakamatsu et al., 2006) and a set of six LAMP primers was used instead of four. The low level of relative specificity of RT-LAMP with cloacal swabs $(33 \%)$ and that with oro-pharyngeal swabs $(67 \%)$ as computed in its comparison with ICA is as a result of poor degree of agreement between the two tests, with RT-LAMP being superior. Like reported in previous similar studies (Pham et al., 2005; Xue et al., 2009; Li et al., 2009), RT-LAMP using clocal and oropharyngeal swabs did not amplify RNA from other viral infections and hence analytical specificity of $100 \%$. The level of relative diagnostic sensitivity and specificity demonstrated by RT-LAMP using cloacal and oro-pharyngeal swabs is a good method for epidemio-logical studies. Generally, at diagnostic sensitivity and specificity of $99 \%$ and $99 \%$, respectively, a test is regarded relatively good for epidemiological studies (Martin et al., 1987).

\section{Conclusion}

Our study has demonstrated that RT-LAMP can detect NDV from cloacal and oro-pharyngeal swabs and in less than 60 minutes with samples amplified using a heat block. The method amplifies RNA from these specimens with high relative sensitivity (98$100 \%)$ and analytical specificity $(100 \%)$, yet results can easily be observed with a naked eye. Use of less invasive samples, the short reaction time, the simple amplification equipment involved, ease of interpretation of results and the high level of analytical and relative sensitivity and specificity make RT-LAMP a good, convenient and inexpensive alternative in diagnosis of NDV in chickens in resource poor countries. Collection of cloacal and oro-pharyngeal swabs does not cause farmer resentment, which makes sample collection easier in different ND outbreaks.

\section{Acknowledgements}

Through National Agricultural Research Organisation (NARO), we thank the Government of the Republic of Uganda for Development Fund (Vote No. 142100000/15101) under which study activities in Uganda were funded. Further appreciation is extended to Japan Society for Promotion of Science Asia-Africa Science Platform Program (Grant No. 0001228) that funded analysis of samples in Japan.

\section{Conflict of interest} interest.

Authors declare that they have no conflict of

\section{References}

1. Alexander, D.J. (2003). Newcastle disease, other paramyxoviruses and Pneumovirus infections. In Saif, Y.M., Barnes, H.J., Glisson, J.R., Fadly, A.M., McDougald, L.R. and Swayne, D.E. (eds) Diseases of poultry (11th edn). Iowa State University 
Press, Ames: 63-99.

2. Anigen (2005). www.anigen.co.kr.com (Accessed 2008).

3. Baba, Y., Hataoka, Y. and Notomi, T. (2002). Integrated Microsystem of isothermal amplification of DNA electrophoresis and microfabricated on a plastic chip for detection of specific gene and analysis of genetic materials: Proceedings of the $6^{\text {th }}$ International Symposium, Micro Total Analysis Systems, Nara, Japan, 3-7 November 2002:215-217.

4. Calnek, B.W., Bernes, H.J., Beard, C.W., Mcdougald, L.R., Saif, Y. M. (1997). Diseases of poultry (10th edn). Iowa State University Press, Ames: 541-605.

5. Fowler, N.G. (1996). "How to carry out a field investigation”. In Jordan, F.T.W., Pattison, W.B. (eds), Poultry Diseases (4th edn). Saunders, London: 422456.

6. Hofstad, M.S., Barnes, H.J., Calnek, B.W., Reid, W.M. and Yoder, H.W. (1984). Diseases of Poultry (8th edn). Iowa State University Press, Ames.

7. IAEA (International Atomic Energy Agency)/FAO (Food and Agriculture Organisation) (2006). Animal Production and Health Newsletter, 44: 8-10.

8. Kommers, G.D., King, D.G., Seal, B.S., Carmichael, K.P. and Brown, C.C. (2002). Pathogenesis of six pigeon-origin isolates of Newcastle disease virus for domestic chickens. Vet. Pathol., 39(3): 353-362, 12014499 Cit: 12

9. Li, Q., Xue, C., Qin, J., Zhou, Q., Chen, F., Bi, Y. and Cao, Y. (2009). An improved reverse transcription loop-mediated isothermal amplification assay for sensitive and specific detection of Newcastle disease virus. Arch. Virol., 154: 1433-1440. DOI: 10.1007/s00705-009-0464-z.

10. Martin, S.W., Alan, H.M. and Willeberg, P. (1987). Veterinary Epidemiology, Principles of Methods (1st edn). Iowa State University Press, Ames.

11. Mori, Y., Nagamine, K., Tomita, N. and Notomi, T. (2001). Detection of loop-mediated isothermal amplification reaction by turbidity derived from magnesium pyrophosphate formation. Biochem Biophys Res Commun., 289: 150-154. doi:10.1006/ bbrc.2001.5921.

12. Motulsky, H. (1995). Intuitive Biostatistics. Oxford University Press.

13. Mukiibi-Muka, G. and Olaho-Mukani, W. (1998) Serological survey of antibodies to infectious bronchitis virus, Newcastle disease virus and mycoplasma in commercial and indigenous chickens in Uganda. Proceedings of the International Symposium on infectious bronchitis and Pneumovirus infection in poultry, (Rauischholzhausen, 1998, World Veterinary Poultry Association). pp: 157 - 165.

14. Nagai, Y., Hamaguchi, M., Toyoda, T. (1989). Molecular biology of Newcastle Disease Virus. Prog Vet Microbiol Immunol., 5: 16-64.

15. Nagamine, K., Hase, T. and Notomi, T. (2002a).
Accelerated reaction by loop-mediated isothermal amplification using loop primers. Mol. Cell. Probes, 16: 223-229. doi:10.1006/mcpr.2002.0415.

16. Nagamine, K., Kuzuhara, K. and Notomi, T.(2002b). Isolation of single-stranded DNA from loop-mediated isothermal amplification products. Biochem. Biophys. Res. Commun., 290: 1195-1198. doi:10.1006/bbrc.2001.6334.

17. Notomi. T, Okayama, H., Masubuchi, H., Yonekawa, T., Watanabe, K., Amino, N. and Hase, T. (2000). Loop- mediated isothermal amplification of DNA Nucl. Acids Res., 28 (12): e63. doi: 10.1093/nar/28.12.e63.

18. OIE (World Organisation for Animal Health) (2010). Newcastle disease. Manual of Diagnostic Tests and Vaccines for Terrestrial Animals, Chapter 2.3.14: pp. 576-589.

19. Okwor, E.C., Okoye, J.O. and Echeonwu, G.O (2007). Distribution of a local isolate of velogenic Newcastle disease virus in organs of infected chickens. Nigerian Veterinary Journal, 28 (3): 19-23.

20. Otim, M.O., Christensen, H., Jorgensen, H.P. Handberg, K.J. and Bisgaard, M. (2004). Molecular characterization and phylogenetic study of Newcastle Disease virus isolates from recent outbreaks in Eastern Uganda. J. Clin. Microbiol. 42(6): 2802-2805. doi: 10.1128/JCM.42.6.2802-2805.2004.

21. Otim, M.O, Kabagambe, E.K., Mukiibi, G.M., Christensen, H. and Bisgaard, M. (2007). A study of risk factors associated with Newcastle disease epidemics in village free-range chickens in Uganda, Trop Anim Health Prod, 39 (1): 27-35. PMID: 17941485.001.

22. Pavlov, A.R., Pavlova, N.V., Kozyavkin, S.A. and Slesarev, A.I. (2004). Recent developments in the optimization of thermostable DNA polymerases for efficient applications. Trends in Biotechnol., 22: 253260. doi:10.1016/j.tibtech.2004.02.011.

23. Pham, H.M., Nakajima, C., Ohashi, K. and Onuma, M. (2005). Loop-mediated isothermal amplification for rapid detection of Newcastle disease virus. J. Clin. Microbiol., 43 (4): 1646-1650.doi:10.1128/JCM. 43.4.1646-1650.2005.

24. Rothbarth, P.H., Groen, J. and Bohnen, A.M. (1999). Influenza virus serology - a comparative study. $J$. Virol. Methods, 78: 163-169. doi:10.1016/S01660934(98)00174-8.

25. Rychlik, W., Spencer, W.J. and Rhoads, R.E. (1990). Optimization of the annealing temperature for DNA amplification in vitro. Nucl. Acids Res., 18 (21): 64096412. doi: 10.1093/nar/18.21.6409.

26. Steel, J., Lowen, A.C., Pena, L., Angel, M., Solórzano, A., Albrecht, R., Perez, D.R., García-Sastre, A. and Palese, P. (2009). Live-attenuated influenza viruses containing NS1 truncations as vaccine candidates against $\mathrm{H} 5 \mathrm{~N} 1$ highly pathogenic avian influenza. $J$ Virol., 83 (4): 1742-1753. doi: 10.1128/JVI.0192008.

27. Tomita, N., Mori, Y., Kanda, H. and Notomi, T 
(2008). Loop-mediated isothermal amplification (LAMP) of gene sequences and simple visual detection of products. Nat. Protoc., 3(5): 877-882. doi:10.1038/nprot.2008.57.

28. Wakamatsu, N., King, D.J., Kapczynski, D.R., Seal, B.S. and Brown, C.C. (2006). Experimental pathogenesis for chickens, turkeys, and pigeons of exotic Newcastle disease virus from an outbreak in California during 2002-2003. Vet. Pathol., 43: 925-933. doi: 10.1354/vp.43-6-925.
29. Xu, H., Lohr., J. and Greiner, M. (1997). The selection of ELISA cut-off points for testing antibody to Newcastle disease by two-graph receiver operating characteristic (TG-ROC) analysis. $J$. Immunol. Methods, 208:61-64.

30. Xue, C., Zhang, Y., Zhou, Q., Xu, C., Li, X. and Cao, Y. (2009). Rapid detection of infectious bursal disease virus by reverse transcription loop-mediated isothermal amplification assay. J. Vet. Diagn. Invest, 21(6):841-843. doi: 10.1177/104063870902100612. 